\title{
IgG4-Associated Cholangitis Can Mimic Hilar Cholangiocarcinoma
}

\author{
Victor M. Zaydfudim*, Andrew Y. Wang ${ }^{\dagger}$, Eduard E. de Lange ${ }^{\ddagger}$, Zimin Zhao ${ }^{\S}$, Christopher A. Moskaluk ${ }^{\S}$, Todd W. Bauer*, \\ and Reid B. Adams* \\ ${ }^{*}$ Section of Hepatobiliary and Pancreatic Surgery, Division of Surgical Oncology, ${ }^{\dagger}$ Division of Gastroenterology and Hepatology, ${ }^{\ddagger}$ Department \\ of Radiology and Medical Imaging, and ${ }^{s}$ Department of Pathology, University of Virginia, Charlottesville, VA, USA
}

IgG4-associated cholangitis can mimic hilar cholangiocarcinoma. Previously reported patients with IgG4-associated cholangitis mimicking cholangiocarcinoma had elevated serum IgG4 levels and long-segment biliary strictures. However, in the absence of other diagnostic criteria for malignancy, IgG4-associated cholangitis should remain a consideration among patients with normal serum IgG4 and a hilar mass suspicious for cholangiocarcinoma. The presence of a hilar mass and a malignant-appearing biliary stricture in two patients with normal serum IgG4 prompted further evaluation and subsequent concomitant liver and bile duct resection and reconstruction. The diagnosis of IgG4-associated cholangitis was established during the pathologic evaluation of the resected specimens. IgG4-associated cholangitis is a known imitator of hilar cholangiocarcinoma and should be considered in the differential diagnosis even among serologically IgG4-negative patients with a hilar mass prior to operative resection. (Gut Liver 2015;9:556-560)

Key Words: IgG4 cholangiopathy; Autoimmune cholangiopathy; Autoimmune pancreatitis; Biliary stricture; Hilar mass

\section{INTRODUCTION}

IgG4-associated cholangitis (IAC) is a recently described biliary manifestation of a systemic autoimmune disorder with a characteristic IgG4-positive histopathologic lymphoplasmacytic infiltrate. ${ }^{1}$ The spectrum of IgG4-related autoimmune disease is broad and includes type 1 autoimmune pancreatitis, as well as sclerosing cholangitis, tubulointerstitial nephritis, sialadenitis, and retroperitoneal fibrosis. The diagnosis and management of autoimmune-mediated pancreatobiliary disorders has evolved dramatically over the past decade. A majority of patients are currently diagnosed using clinically suspicious cross-sectional imaging, elevated IgG4 serum levels, and/or IgG4-positive cells in the biopsy specimens. ${ }^{1,2}$

The spectrum of IgG4-associated cholangitis commonly includes intra- and extrapancreatic biliary strictures. A majority of the patients have concomitant type 1 autoimmune pancreatitis and elevated serum IgG4 levels. Diagnosis can be frequently established by clinical suspicion and elevated serum IgG4. Empiric steroid therapy can help to confirm the diagnosis if disease remission occurs. A minority of patients with isolated biliary strictures have been treated with operative resection for presumed cholangiocarcinoma. ${ }^{1,34}$

The diagnosis of hilar cholangiocarcinoma can be elusive. The presence of a malignant appearing stricture and corresponding mass lesion on cross-sectional imaging is sufficient for the diagnosis of hilar cholangiocarcinoma., ${ }^{5,6}$ Transperitoneal biopsies and endoscopic ultrasound-directed aspiration of suspicious tumor are avoided to minimize the risk of postoperative recurrence. ${ }^{6,7}$ We describe two patients with normal serum IgG4 levels and a mass at the biliary hilum with a corresponding malignantappearing hilar stricture. In each case, the clinical presentation and imaging findings were consistent with hilar cholangiocarcinoma; both of the patients were treated with hemihepatectomy, radical bile duct resection, hepatoduodenal ligament lymphadenectomy and biliary reconstruction. Histopathologic evaluation of the resected mass in both patients revealed IgG4-associated cholangitis. In this report, we discuss the clinical evaluation and treatment of the two patients with this diagnosis and propose alternative adjuncts to the diagnostic evaluation and management to help reduce the risk of an unnecessary operation.

\section{CASE REPORTS}

\section{Case 1}

A 68-year-old man presented with nausea and abdominal

Correspondence to: Victor M. Zaydfudim

Section of Hepatobiliary and Pancreatic Surgery, Division of Surgical Oncology, University of Virginia, Box 800709, Charlottesville, VA 22908, USA

Tel: +1-434-924-2839, Fax: +1-434-982-4778, E-mail: vz8h@virginia.edu

Received on July 3, 2014. Accepted on July 24, 2014. Published online June 4, 2015

pISSN 1976-2283 eISSN 2005-1212 http://dx.doi.org/10.5009/gnl14241

() This is an Open Access article distributed under the terms of the Creative Commons Attribution Non-Commercial License (http://creativecommons.org/licenses/by-nc/4.0) which permits unrestricted non-commercial use, distribution, and reproduction in any medium, provided the original work is properly cited. 
pain. Initial studies demonstrated mild transaminitis (aspartate aminotransferase [AST], $51 \mathrm{U} / \mathrm{L}$; alanine aminotransferase [ALT], $65 \mathrm{U} / \mathrm{L})$. Alkaline phosphatase was also slightly elevated at 223 $\mathrm{U} / \mathrm{L}$; total bilirubin was normal at $0.6 \mathrm{mg} / \mathrm{dL}$. Initial imaging suggested intrahepatic biliary dilatation. Subsequent magnetic resonance imaging (MRI)/magnetic resonance cholangiopancreatography (MRCP) demonstrated a 1.0-cm hilar mass (Fig. 1A) with a corresponding hilar stricture and intrahepatic biliary obstruction (Fig. 1B), concerning for Bismuth-Corlette IIIA hilar cholangiocarcinoma. The patient did not have evidence of pancreatitis. The IgG panel was normal; the IgG4 level was 61.3 $\mathrm{mg} / \mathrm{dL}$ (normal range, 2.4 to $121 \mathrm{mg} / \mathrm{dL}$ ). CA 19-9 level was 41 $\mathrm{U} / \mathrm{mL}$ (normal, <37 U/mL). Bile duct brushings demonstrated atypical ductal cells suspicious for adenocarcinoma. The patient underwent a right hepatectomy with hepatoduodenal lymphadenectomy, radical bile duct resection, and Roux-en-Y hepaticojejunostomy.

\section{Case 2}

A 79-year-old man was referred for the evaluation of incidental liver function test abnormalities on routine laboratory stud- ies. Initial investigations revealed elevations in the AST (73 U/ L), ALT (80 U/L), and alkaline phosphatase (430 U/L) levels. The total bilirubin was normal at $0.5 \mathrm{mg} / \mathrm{dL}$. Initial imaging showed intrahepatic biliary dilatation. MRI/MRCP identified a $2.1-\mathrm{cm}$ hilar mass (Fig. 2A), with a corresponding hilar stricture and intrahepatic biliary obstruction (Fig. 2B), concerning for BismuthCorlette IIIA hilar cholangiocarcinoma. The patient did not have evidence of pancreatitis. The IgG4 level was normal at 39.4 $\mathrm{mg} / \mathrm{dL}$; the CA 19-9 level was $4 \mathrm{U} / \mathrm{mL}$. Bile duct brushings did not reveal suspicious biliary cells. Given the presence of a hilar mass and a corresponding malignant-appearing biliary stricture, resection was planned. The patient underwent right portal vein embolization to augment future liver remnant, followed by right hepatectomy with hepatoduodenal lymphadenectomy, radical bile duct resection, and Roux-en-Y hepaticojejunostomy.

Histopathologic evaluation of both of the specimens revealed a dense mucosal lymphoplasmacytic infiltrate, transmural aggregates of chronic inflammatory cells, and dense fibrosis of the wall of the common bile ducts, with no evidence of malignancy (Fig. 3A). Fibrosis consisted of fibrillary or thick collagen bands with spindled fibroblasts or myofibroblasts (Fig. 3B). The lym-
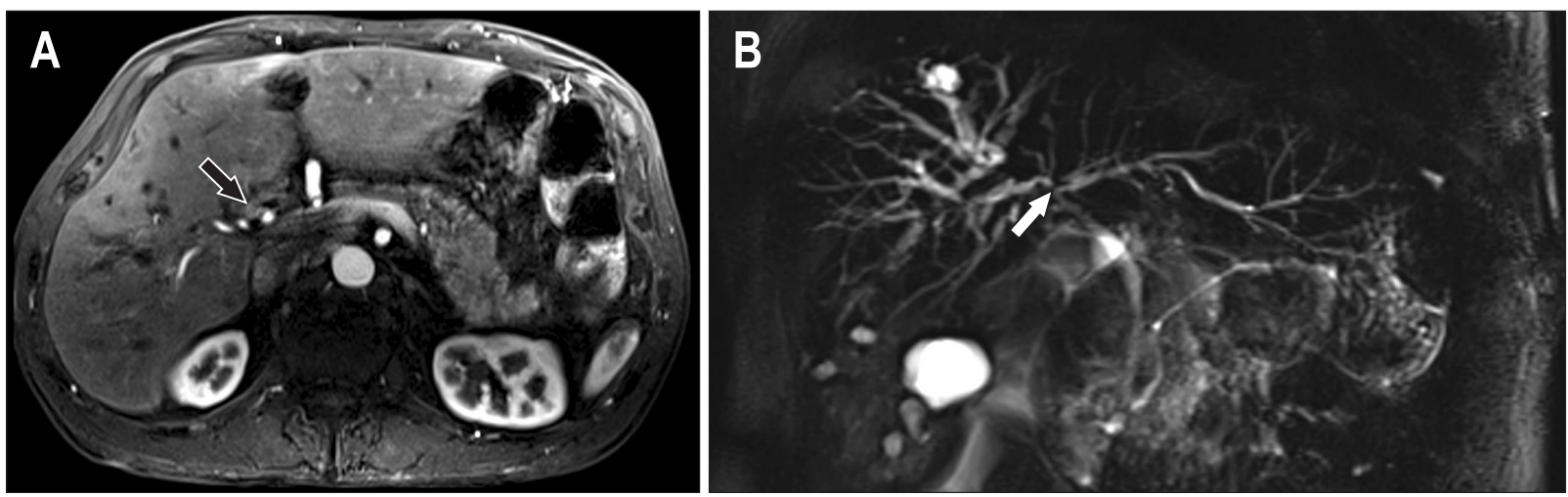

Fig. 1. MR contrast-enhanced T1-weighted image (A) demonstrating a 1-cm hilar mass (arrow) with a MR cholangiogram (B) demonstrating a corresponding hilar biliary stricture (arrow).
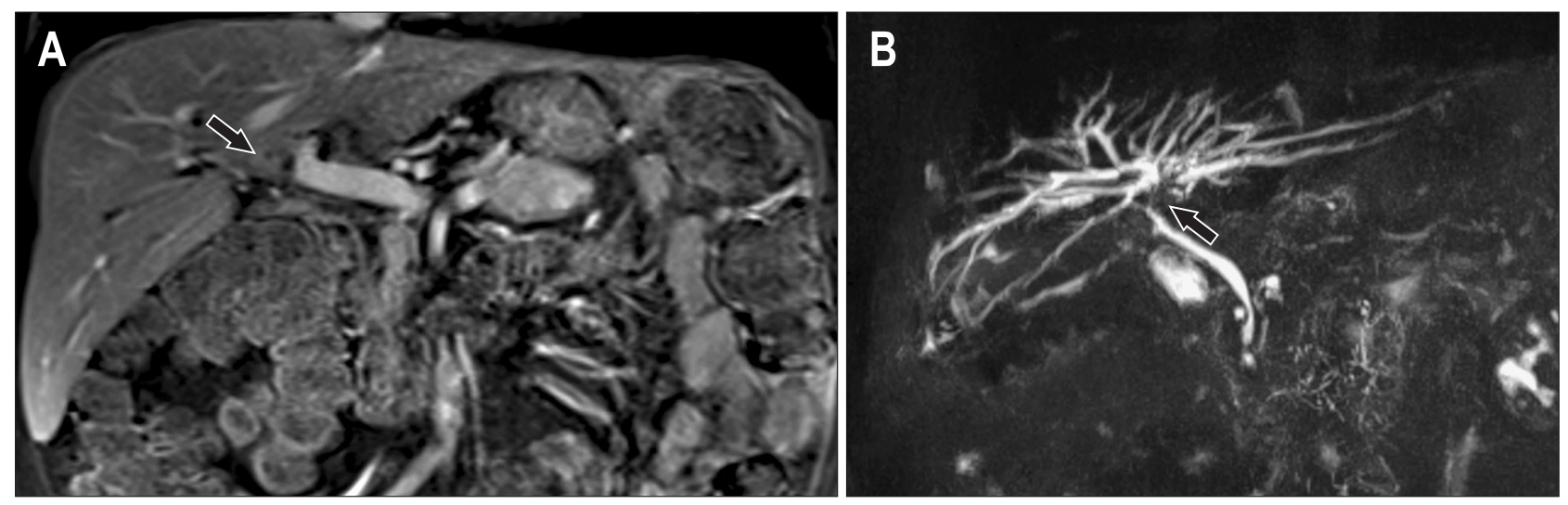

Fig. 2. MR contrast-enhanced T1-weighted image (A) demonstrating a 2.1-cm hilar mass (arrow) with a MR cholangiogram (B) demonstrating a corresponding hilar biliary stricture (arrow). 

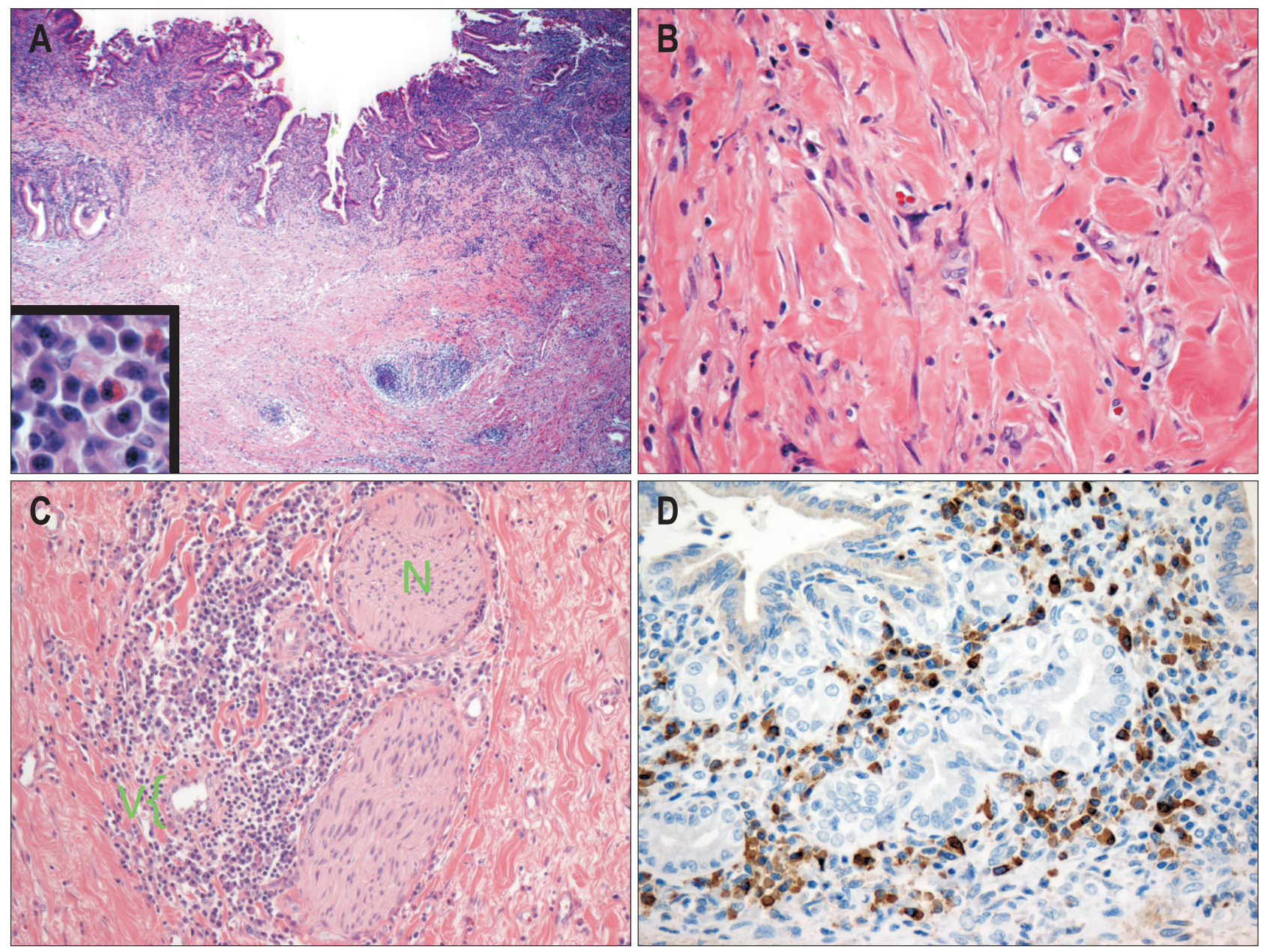

Fig. 3. Histologic features of IgG4-associated cholangitis. (A) Common bile duct with dense mucosal inflammation, transmural inflammation and fibrosis (H\&E stain, $\times 20$; inset: magnification of mucosal inflammatory infiltrate, showing the predominance of plasma cells with occasional eosinophils, $\times 400$ ). (B) Fibrosis of the common bile duct wall. Thick collagen bundles are present with intervening spindled fibroblasts and lymphocytes (H\&E stain, $\times 200)$. (C) Neurovascular bundle within the common bile duct wall with lymphoplasmacytic infiltration of a nerve (N) and a small vein (V) (H\&E stain, $\times 100)$. (D) Immunohistochemical stain for IgG4, demonstrating a high density of IgG4-positive plasma cells (brown stain) in the common bile duct mucosa $(\times 200)$.

phoplasmacytic infiltrates were frequently condensed around neurovascular bundles, involving the venous wall and the perineurium (Fig. 3C). There was relative sparing of accompanying arteries from the infiltrate compared to the venous channels. Occasional small germinal center formation was observed in one of the two cases. A small to moderate number of eosinophils were also present, admixed within the inflammatory infiltrates.

Immunohistochemical staining for IgG4 and CD138 showed that greater than $40 \%$ of the plasma cells were IgG4 positive, with multiple areas showing greater than 50 IgG4-positive plasma cells per high-power field (Fig. 3D), characteristic of IgG4associated cholangitis.

\section{DISCUSSION}

Resection is the only chance for cure in patients with hilar cholangiocarcinoma. Because hilar cholangiocarcinoma can be notoriously difficult to pathologically diagnose prior to resection, clinical diagnosis requires the presence of a malignantappearing biliary stricture and at least one of the following: (1) a mass lesion on cross-sectional imaging at the location of the malignant-appearing stricture; (2) endoluminal biopsy or cytology positive for cholangiocarcinoma; (3) polysomy by fluorescent in situ hybridization; or (4) CA 19-9 >100.,6 Both of the patients in this series originally presented with a hilar mass and a corresponding malignant-appearing biliary stricture. One of the two patients also had biliary brushings suspicious for adenocarcinoma.

However, a number of features in the clinical presentation of these two patients were not unequivocally consistent with cholangiocarcinoma. Biliary obstruction, jaundice, and hyperbilirubinemia are the most common initial clinical features of patients with hilar cholangiocarcinoma, but approximately 10\% of patients present without symptoms. ${ }^{8,9}$ Both of the patients in 
our series presented without clinical or biochemical evidence of biliary obstruction and normal CA 19-9 levels. The sensitivity of elevated CA 19-9 for cholangiocarcinoma is only $40 \%$ to $70 \%$, the specificity is $50 \%$ to $80 \%$, and the positive predictive value is $16 \%$ to $40 \%{ }^{9,10}$ A study evaluating the utility of CA 19-9 for the diagnosis of cholangiocarcinoma in patients without primary sclerosing cholangitis (PSC) estimated that 47\% of the patients had cholangiocarcinoma with CA 19-9 levels $<100$ $\mathrm{U} / \mathrm{mL} .^{10}$ Similarly, endobiliary brush cytology and endoscopic transpapillary biopsy also lack sensitivity for the diagnosis of cholangiocarcinoma. ${ }^{11,12}$

In contrast, the clinical suspicion for IgG4-associated cholangitis was low in both patients. Previous studies described a high association between IAC and symptoms of obstructive jaundice, autoimmune pancreatitis, and increased serum IgG4 levels. ${ }^{1}$ These features were absent in our patients. While we evaluated biliary brushings for cellular atypia, bile duct biopsies were not performed in either patient. Reports discussing the clinical utility of endobiliary bile duct biopsies for the diagnosis of IAC are mixed, with a range of IgG4-positive lymphoproliferative infiltrates (defined as more than 10 IgG4-positive cells per HPF) described in $18 \%$ to $88 \%$ of the patients. ${ }^{13-15}$ Up to $10 \%$ of patients with preoperatively presumed cholangiocarcinoma and no evidence of IAC who underwent resection at experienced hepatobiliary centers are diagnosed with nonmalignant biliary pathology after review of the resected specimen. ${ }^{16}$

Can more extensive preoperative evaluation prevent unnecessary resection in patients with undiagnosed IAC and normal serum IgG4 levels? One attractive option appears to be the measurement of IgG4 levels in bile. A recent report found markedly elevated IgG4 levels in patients with IAC compared to patients with cholangiocarcinoma, PSC, or biliary stone disease. ${ }^{4}$ The differences in the IgG4 bile measurements between IAC and other pathologies are significant, with mean IgG4 levels of $41 \mathrm{mg} / \mathrm{dL}$ among patients with IAC versus $<3 \mathrm{mg} / \mathrm{dL}$ among patients with non-IgG4 mediated biliary disease. Another option is the use of selective endobiliary bile duct biopsies to diagnose IAC. With the highly variable published data already discussed, we have not routinely tested preresection endoscopic bile duct samples for histopathologic IgG4-positive infiltrates. However, while the sensitivity varies between $18 \%$ and $88 \%$, the specificity of $>10$ IgG4-positive cells per HPF in patients with IAC approaches $100 \%{ }^{15}$ As such, selective evaluation of endobiliary bile duct samples for IgG4-rich plasma cells should be considered when technically feasible for patients with biliary strictures and a possibility of IAC who do not have a definitive diagnosis of cholangiocarcinoma.

Finally, empiric steroid treatment can be used to distinguish IAC from other causes of biliary strictures. A steroid trial was recently described that distinguished patients with autoimmune pancreatitis (but without other diagnostic criteria) from those who had pancreatic adenocarcinoma. ${ }^{17}$ Five of the 48 patients in that series were diagnosed with AIP based on a response to empiric steroid treatment. Any empiric steroid treatment for suspected IAC, however, should be performed selectively and with close follow-up. One of two patients presented in our series had cytologic biliary atypia indicating adenocarcinoma. It is difficult to speculate whether additional evaluation would have precluded resection in that patient.

IgG4-associated cholangitis is known to mimic hilar cholangiocarcinoma. We present two patients with a combination of a hilar mass and a malignant-appearing biliary stricturetypically sufficient diagnostic criteria to support resection for suspected hilar cholangiocarcinoma. Both of these patients presented without associated jaundice or elevated CA 19-9 levels. While serologic and radiographic evidence did not suggest the presence of IgG4-related autoimmune disease, additional diagnostic evaluation could have revealed evidence of IAC. In patients with this pattern of findings and persistent diagnostic uncertainty, additional diagnostic maneuvers to diagnose IgG4associated cholangitis should be considered prior to resection of suspected hilar cholangiocarcinoma.

\section{CONFLICTS OF INTEREST}

No potential conflict of interest relevant to this article was reported.

\section{REFERENCES}

1. Ghazale A, Chari ST, Zhang L, et al. Immunoglobulin G4-associated cholangitis: clinical profile and response to therapy. Gastroenterology 2008;134:706-715.

2. Kamisawa T, Chari ST, Lerch MM, Kim MH, Gress TM, Shimosegawa $\mathrm{T}$. Recent advances in autoimmune pancreatitis: type 1 and type 2. Gut 2013;62:1373-1380.

3. Hamano H, Kawa S, Uehara T, et al. Immunoglobulin G4-related lymphoplasmacytic sclerosing cholangitis that mimics infiltrating hilar cholangiocarcinoma: part of a spectrum of autoimmune pancreatitis? Gastrointest Endosc 2005;62:152-157.

4. Vosskuhl K, Negm AA, Framke T, et al. Measurement of IgG4 in bile: a new approach for the diagnosis of IgG4-associated cholangiopathy. Endoscopy 2012;44:48-52.

5. Rosen CB, Heimbach JK, Gores GJ. Liver transplantation for cholangiocarcinoma. Transpl Int 2010;23:692-697.

6. Zaydfudim VM, Rosen CB, Nagorney DM. Hilar cholangiocarcinoma. Surg Oncol Clin N Am 2014;23:247-263.

7. Heimbach JK, Sanchez W, Rosen CB, Gores GJ. Trans-peritoneal fine needle aspiration biopsy of hilar cholangiocarcinoma is associated with disease dissemination. HPB (Oxford) 2011;13:356-360.

8. de Groen PC, Gores GJ, LaRusso NF, Gunderson LL, Nagorney DM. Biliary tract cancers. N Engl J Med 1999;341:1368-1378.

9. Khan SA, Davidson BR, Goldin RD, et al. Guidelines for the diagnosis and treatment of cholangiocarcinoma: an update. Gut 
2012;61:1657-1669.

10. Patel AH, Harnois DM, Klee GG, LaRusso NF, Gores GJ. The utility of CA 19-9 in the diagnoses of cholangiocarcinoma in patients without primary sclerosing cholangitis. Am J Gastroenterol 2000; 95:204-207.

11. Barr Fritcher EG, Kipp BR, Slezak JM, et al. Correlating routine cytology, quantitative nuclear morphometry by digital image analysis, and genetic alterations by fluorescence in situ hybridization to assess the sensitivity of cytology for detecting pancreatobiliary tract malignancy. Am J Clin Pathol 2007;128:272-279.

12. Razumilava N, Gores GJ. Classification, diagnosis, and management of cholangiocarcinoma. Clin Gastroenterol Hepatol 2013;11: 13-21.e1.

13. Naitoh I, Nakazawa T, Ohara H, et al. Endoscopic transpapillary intraductal ultrasonography and biopsy in the diagnosis of IgG4related sclerosing cholangitis. J Gastroenterol 2009;44:1147-1155.

14. Kawakami H, Zen Y, Kuwatani M, et al. IgG4-related sclerosing cholangitis and autoimmune pancreatitis: histological assessment of biopsies from Vater's ampulla and the bile duct. J Gastroenterol Hepatol 2010;25:1648-1655.

15. Zen Y, Nakanuma Y. IgG4 Cholangiopathy. Int J Hepatol 2012; 2012:472376.

16. Baskin-Bey ES, Devarbhavi HC, Nagorney DM, et al. Idiopathic benign biliary strictures in surgically resected patients with presumed cholangiocarcinoma. HPB (Oxford) 2005;7:283-288.

17. Chari ST, Takahashi N, Levy MJ, et al. A diagnostic strategy to distinguish autoimmune pancreatitis from pancreatic cancer. Clin Gastroenterol Hepatol 2009;7:1097-1103. 